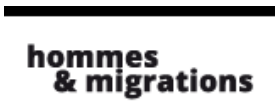

\section{Hommes \& migrations}

Revue française de référence sur les dynamiques

migratoires

$1314 \mid 2016$

Migrations chinoises et générations

\title{
Moving Beyond Calais
}

\section{Cécilia Fall}

\section{(Q) OpenEdition \\ Journals}

\section{Édition électronique}

URL : https://journals.openedition.org/hommesmigrations/3649

DOI : 10.4000/hommesmigrations.3649

ISSN : 2262-3353

\section{Éditeur}

Musée national de l'histoire de l'immigration

\section{Édition imprimée}

Date de publication : 1 avril 2016

Pagination : 120-123

ISBN : 978-2-919040-35-3

ISSN : $1142-852 X$

\section{Référence électronique}

Cécilia Fall, « Moving Beyond Calais », Hommes \& migrations [En ligne], 1314 | 2016, mis en ligne le 19 septembre 2016, consulté le 28 juin 2022. URL : http://journals.openedition.org/hommesmigrations/ 3649 ; DOI : https://doi.org/10.4000/hommesmigrations.3649 


\section{SPÉCIAL FRONTIÈRES}

\section{MOVING BEYOND CALAIS}

Par CÉCILIA FALL, volontaire Migreurop au sein de la Plateforme de Service aux Migrants.

A u début de l'année 2015, la mairie de Calais ouvre le centre d'accueil de jour Jules Ferry. Une amélioration ? Pas vraiment. Le centre ne propose qu'un repas par jour, 60 douches et un accueil de nuit pour 400 femmes et enfants. En conséquence, des milliers de personnes continuent de vivre dans des lieux insalubres. Par ailleurs, elle somme les exilé(e)s qui vivent dans la ville de Calais, de se déplacer sur le terrain qui jouxte ce centre. Les squats et campements du centre-ville de Calais et ses environs seront évacués et détruits - parfois dans la violence - les uns après les autres. À ce jour, ce sont environ 6 ooo personnes qui survivent sur ce camp (appelé "La Lande" par les autorités), un terrain qui appartient pour moitié à la mairie et pour l'autre à la région Nord-Pas-de-Calais, classé SEVESO et non constructible. Invisibilisés et relégués en dehors de la ville, concentrés sur ce seul espace, à deux pas du port de Calais, principal point de passage vers le Royaume-Uni, mais à $14 \mathrm{~km}$ de l'Eurotunnel, autre point de passage, ce camp s'est transformé petit-à-petit en bidonville avec ses restaurants, magasins et lieux de vie sociale, culturelle et religieuse. La médiatisation de ce que l'on appelle "la crise des réfugiés" au cours de cette même année a aussi contribué à un élan de solidarité, concentré sur le camp de Calais : plusieurs centaines de bénévoles, associatifs ou indépendants, issus de différents pays européens, s'activent à pallier l'indifférence dont fait preuve l'État français, et répondre aux besoins fondamentaux des exilé(e)s : nourriture, abris, tentes, vêtements, mais aussi culture et cours de français.

Les conditions de vie demeurent néanmoins terribles pour le grand nombre de personnes qui continuent de transiter par Calais et les villes environnantes (Grande-Synthe, Norrent-Fontes, etc.). En conséquence, face aux conditions inhumaines et dégradantes auxquelles les exilé(e)s ne cessent d'être confrontés, l'urgence continue d'être le principal mode d'action pour les associations, les bénévoles présents en permanence ainsi que celles et ceux de passage quelques jours ou quelques semaines. À tel point, qu'il est facile d'oublier les causes de cette situation migratoire que de nombreux responsables politiques en France, au Royaume-Uni voire au sein de l'Union européenne, maintiennent faute de courage politique. Cela contribue à éloigner aussi ces acteurs "humanitaires" les uns des autres : chacun répond aux urgences qu'il voit et dont il a connaissance. Mais il y a 6 ooo urgences.

Prendre le temps de se rencontrer, de se connaître, mais aussi de réfléchir collectivement, constitue une autre urgence à laquelle répond Moving Beyond Borders (MBB), exposition itinérante produite par le réseau Migreurop et mise en scène par la compagnie Étrange Miroir. Cette exposition s'est tenue durant la rencontre internationale : “'Hotspots' et 'processing centres' : Les nouveaux 
17 décembre 2015, elle a été accueillie à la Bibliothèque universitaire de l'université de la Côte d'Opale de Calais. Tout au long de cette seconde étape qui a duré deux semaines, différentes médiations ont été proposées. L'idée de ces "visites guidées" était à la fois d'expliquer les conséquences des politiques migratoires actuelles sur les parcours des exilé(e)s mais aussi d'établir des liens entre les réalités dévoilées par l'exposition et la situation régionale du Nord-Pas-de-Calais. Les associations qui interviennent auprès des exilé(e)s de cette région ainsi que des bénévoles, ont été associé(e)s à cette démarche pour partager leurs expériences et leur expertise.

\section{Des visites scolaires : informer et expliquer}

Plusieurs groupes scolaires ont visité l'exposition et partagé un moment de discussion

habits de la politique européenne d'encampement, d'externalisation et de tri des exilé(e)s", qui a permis à certains acteurs intervenant à Calais et dans son bidonville de se rencontrer et d'échanger.

\section{La culture, comme vecteur de rencontre et "antidote" à la peur de l'Autre}

L'exposition Moving Beyond Borders, qui propose une approche multimédia - créative et inédite - des réalités migratoires, explique la façon dont les politiques européennes impactent sur les parcours des exilé(e)s. À travers des cartographies dessinées et animées, des œuvres se construisent sous les yeux des spectateurs. Agrémentées de bandes audio et de photographies, l'exposition est le fruit d'un collectif regroupant une quarantaine de personnes. Après son inauguration en juin 2015 à Bruxelles, du 2 au avec des bénévoles et militants associatifs. La recherche d'un apport purement informatif, loin de toute dichotomie entre les personnes perçues comme "pro-migrant(e)s" et "anti-migrant(e)s", a été clairement exprimée par certains élèves et étudiants. En lien avec l'exposition, les discussions se sont centrées sur les causes d'une telle situation au sein de la région. Cela a ensuite permis la déconstruction de certains préjugés sur les conditions de vie des exilé(e)s ou de leur parcours migratoire.

\section{Encourager et alimenter le questionnement}

Bien souvent, l'intervention d'une personne extérieure à ces vies itinérantes et à l'action des organisations favorise la prise de distance avec les enjeux auxquels chacun des acteurs doit faire face, la plupart du temps dans l'urgence. Les différents acteurs intervenant sur les lieux de 


\section{SPÉCIAL FRONTIÈRES}

(sur)-vie des exilé(e)s ne prennent, en effet, pas toujours le temps de se rencontrer, concentrés sur leurs propres actions. Le passage de MBB fut, pour certains, l'occasion d'échanger. Accompagnés de la chercheuse Mathilde Pette, des militants associatifs de Norrent-Fontes et du Havre confrontés à des réalités à la fois différentes et similaires ont partagé leurs questionnements autour de MBB. L'ONG Médecins Sans Frontières a pu présenter ses différentes activités à la fois dans la région mais également en Europe. Tout cela, en apportant son expertise sur les enjeux de l'accès à la santé des personnes en migrations, tout au long de leur voyage. Ce, d'autant plus, que depuis quelques mois, le bidonville de Calais et les autres campements de la région où vivent des milliers de personnes sont sous les feux des projecteurs médiatiques. II est donc essentiel de réfléchir à la manière dont nous en parlons, comment nous représentons ces lieux de (sur) vie des exilé(e)s et ce que nous voulons leur faire dire. Louise Druelle, dessinatrice, et Haydé Sabéran, journaliste, sont aussi venues partager leurs expériences et analyses de la situation migratoire de Calais et sa région, à travers divers textes, croquis et dessins.

\section{Susciter la rencontre}

De cette exposition, sont donc nées des rencontres et des dialogues. Au sein de l'université, lieu d'apprentissage et de savoir, des rencontres entre "personnes vulnérables" que des modes de (non)prise en charge tiennent à distance, des habitants et des visiteurs venus d'ailleurs se sont produites le temps d'une visite. Des personnes en situation de handicap ont aussi pu rencontrer de jeunes mineurs isolés étrangers (MIE) et faire leur connaissance, et vice-versa. Ces dialogues autour de jeux du monde construits par ces jeunes MIE et issus du projet La Saveur de l'Autre (mai 2015 au Channel, scène nationale de Calais) ont permis de déconstruire de nombreux préjugés et peurs réciproques. Comme de nombreux migrant(e)s, cette exposition a depuis continué sa route. Elle a fait étape en janvier-février 2016 à Anis Gras (Arcueil), du 10 au 14 mai à Pozzallo (Italie) dans le cadre du festival "Sabir" et se tient du 14 juin au 2 juillet à Poitiers pour les 30 ans du laboratoire de recherche Migrinter. En novembre, elle traversera la Méditerranée pour aller à Rabat (Maroc). De son côté, la Bibliothèque universitaire de Calais poursuit la mise en place des moments de rencontre entre étudiants, jeunes hébergés en maison d'enfants, exilés - jeunes et moins jeunes - des jungles du Calaisis et habitants de la région, tout en continuant d'accueillir des expositions comme Le droit de vivre d'Isabelle Serro. I 